\title{
Inflammatory cytokine IL-8/CXCL8 promotes tumour escape from hepatocyte-induced dormancy
}

\author{
Ahmad S Khazali ${ }^{1}$, Amanda M Clark ${ }^{1}$ and Alan Wells ${ }^{\star, 1,2,3}$ \\ ${ }^{1}$ Department of Pathology, University of Pittsburgh, Pittsburgh, PA 15261, USA; ${ }^{2}$ Pittsburgh VA Health System, Pittsburgh, \\ PA 15213, USA and ${ }^{3}$ University of Pittsburgh Cancer Institute, Pittsburgh, PA 15232, USA
}

Background: Breast cancers can recur after a long latency period following 'successful' primary treatments. Chronic inflammation significantly correlates with reduced diseased-free survival in breast cancer patients and could be a point of intervention to prevent recurrence. Liver is among the main sites of breast cancer recurrence. Thus, we hypothesise that inflammatory signals from hepatic stellate cells, the major inflammatory regulators in the sinusoid, could stimulate dormant cancer cells to emerge.

Methods: We utilise in vitro co-culture of breast cancer cells with stellate cells and an ex vivo 3D human liver micro-physiologic system to identify stellate cells-derived factors that mediate tumour emergence.

Results: Activated, but not quiescent, hepatic stellate cells secreted soluble factors to induce the proliferation of MCF7 and MDAMB231 cancer cells. IL-8 and MCP-1 were highly secreted by the activated stellate cells and primary human non-parenchymal cells. IL-8 significantly reduced serum-starvation growth arrest on MDA-MB231 cells in vitro and increased cancer proliferation ex vivo. Blocking IL-8Rb/CXCR2 reduced IL-8-induced cancer growth and proliferation.

Conclusions: Activated stellate cells can induce breast cancer emergence from dormancy in the liver by secreting inflammatory cytokines. Preventing liver inflammation or disrupting the subsequent key cytokines may prevent metastatic outgrowth.

Breast cancer is a major health problem in the US and worldwide. Although significant progresses have been made in primary breast cancer treatment, long-term survival and breast cancer-related mortality remain poor due to late cancer recurrence and metastasis (Siegel et al, 2017). Accordingly, more than a third of the patients will suffer recurrence at various time points post primary treatment with the majority of the late recurrences are metastatic outgrowths (Lee, 1985; Karrison et al, 1999).

Late recurrences are due to dormant cancer cells that remain asymptomatic and undetectable following dissemination. These dormant micrometastases are particularly difficult to treat since these cells are most likely in a state of non- or intermittent proliferation (Taylor et al, 2013), thus rendering them insensitive to chemotherapy. Breast cancer cells mainly metastasise to the liver, lung, bone and brain. Patients with liver metastases typically show poorer prognosis when compared with bone and lung metastases (Tabariès and Siegel, 2011). Experimentally, we have previously reported that normal liver cells can impose a reversion of the cancer-related epithelial-mesenchymal transition (EMT) back to epithelial phenotype (Chao et al, 2010). E-cadherin reexpression results in smaller tumour nodules and resistance against chemotherapy-induced apoptosis ex vivo (Chao et al, 2012) and in vivo ( $\mathrm{Ma}$ et al, 2016). These results suggest that cancer cells may undergo dormancy in the liver. Preventing tumour outgrowth could be a viable alternative approach to shift metastatic cancer from a largely fatal disease to a chronic disease. Thus, the signals or events that drive emergence from dormancy are keys to lengthening survival.

*Correspondence: Dr A Wells; E-mail: wellsa@upmc.edu

Received 14 September 2017; revised 17 October 2017; accepted 20 October 2017; published online 23 November 2017

(C) 2018 Cancer Research UK. All rights reserved 0007 - 0920/18 
Earlier studies show that thrombospondin 1 and tissue factor can induce tumour cells to undergo dormancy whereas transforming growth factor $\beta 1$, periostin and the interaction between urokinase plasminogen activator receptor and $\alpha 5 \beta 1$ integrin can promote tumour outgrowth (Aguirre-Ghiso et al, 2003; Ghajar et al, 2013; Magnus et al, 2014). Although these studies have shed some light on the mechanistic understandings of tumour emergence, the lack of suitable experimental model impedes research on tumour dormancy in the liver. In an all-human ex vivo 3D liver microphysiological system (MPS), we demonstrated spontaneous dormancy in a subpopulation $(\sim 35 \%)$ of the cells (Wheeler et al, 2014; Clark et al, 2017). However, the signals that drive tumour emergence from dormancy in the liver are still unclear though there are indications that inflammation is contributory (Clark et al, 2017).

Liver microenvironment consists of several different types of cells. Both hepatic parenchyma, hepatocytes, and hepatic nonparenchymal cells (NPCs) such as liver sinusoidal endothelial cells (LSECs), Kupffer cells and hepatic stellate cells (HSCs) play pivotal roles in liver physiology and pathology. Our previous work showed that co-culture with human primary NPC promoted the growth of breast cancer cells when compared to co-culture with hepatocytes alone (Taylor et al, 2014). We further showed that stressed LSECs promoted tumour growth through epidermal growth factor receptor activation (Taylor et al, 2014), consistent with the finding of outgrowth of small metastases at the tips of angiogenic sprouts (Ghajar et al, 2013). Kupffer cells, the liver resident macrophages, displayed phenotype plasticity where the alternative M2 phenotype significantly augmented tumour growth (Yang et al, 2016). Herein, we queried the role of human HSC in tumour growth and emergence in human 3D liver MPS.

HSCs are stromal cells that uptake and store vitamin A in their non-activated state in normal livers (Friedman, 2008). Upon liver injury or infection, the HSCs transdifferentiate into myofibroblast, actively secrete collagen matrix and secrete a plethora of chemokines/cytokines, thus linking injury to fibrosis and chronic inflammation that create a suitable setting for cancer outgrowth. Recent findings suggested that the HSCs are the major inflammatory regulators in the sinusoids (Fujita et al, 2016). We posited that these inflammatory cascades can lead to tumour escape from dormancy in the liver.

Here, we first observed that the activated HSCs elevated breast cancer cell growth and proliferation in vitro. We also found that activated primary human NPC and activated HSCs secreted high level of pro-inflammatory factors including interleukin-8 (IL-8) and monocyte chemoattractant protein-1 (MCP-1). In vitro, IL-8 significantly enhanced MDA-MB231 survival under serum starvation, potentially via ERK activation. Importantly, we also showed that IL-8 could mediate tumour emergence from dormancy in the 3D liver MPS.

\section{MATERIALS AND METHODS}

Cell culture. RFP-labelled MDA-MB231 and MCF7 cells were maintained in RPMI-1640 Glutamax (Gibco) supplemented with $10 \%$ FBS, $1 \times$ penicillin/streptomycin (Gibco) and puromycin $(5 \mu \mathrm{g}$ for MDA-MB231 and $1 \mu \mathrm{g}$ for MCF7). Human mammary epithelial cells (HMEC-1) were cultured in mammary epithelial growth medium (Lonza). Human HSC lines (LX-1 and LX-2) and rat HSC line (HSC-T6), gifts from Dr Scott Friedman (Icahn School of Medicine at Mount Sinai, NY, USA), were cultured in $4.5 \mathrm{gl}^{-1}$ of glucose DMEM without sodium pyruvate (Lonza) supplemented with $10 \%$ FBS $(2 \%$ FBS for LX-2) and $1 \times$ penicillin/ streptomycin. TWNT-1 (human HSC line) and TMNK-1 (human endothelial cell line) cells, gifts from Dr Alex Soto-Gutierrez
(University of Pittsburgh, PA, USA), were cultured in $4.5 \mathrm{gl}^{-1}$ of glucose DMEM with L-glutamine and sodium pyruvate (Corning) supplemented with $10 \% \mathrm{FBS}$ and $1 \times$ penicillin/streptomycin.

Co-culture experiments. LX-1 and LX-2 monolayers were seeded in complete DMEM $+10 \%$ FBS medium. The next day, HMEC-1 cells were labelled with $10 \mu \mathrm{M}$ CMTPX CellTracker (Molecular Probes) for $45 \mathrm{~min}$ prior to co-culture with LX-1 or LX-2 cells. RFP-labelled cancer cells or CMTPX-labelled normal cells were seeded onto HSC monolayer in serum-free hepatocyte maintenance medium (HMM) for LX-1 co-culture or in DMEM $+0.2 \%$ dialysed FBS for LX-2 co-culture. The samples were imaged for RFP signals $4-6 \mathrm{~h}$ post-seeding (day 0 ). On day 2 , the media were exchanged and $20 \mu \mathrm{M}$ of EdU (Invitrogen) were added. On day 4, the samples were fixed with $4 \%$ paraformaldehyde (Electron Microscopy Sciences) and imaged for RFP signals at the same fields of day 0 . RFP percent (RFP\%) area was quantified using ImageJ and normalised to day 0 for growth fold-change. EdU staining was performed according to the manufacturer's protocol. Samples were imaged for RFP, EdU-Alexa Fluor 488 (Invitrogen) and DAPI (as a counterstain) from at least three random fields of view. The number of EdU-positive cells was counted using ImageJ and plotted as a percentage to the total RFP/CMTPX-positive cells counted.

Transwell assay and CXCR2 neutralisation. LX-1 cells were seeded in transwell inserts and cultured overnight. RFP-labelled cancer cells were seeded in 24-well plates and cultured with LX-1 transwell insert in HMM. CXCR2 $\left(1 \mu \mathrm{g} \mathrm{ml}^{-1}\right)(\mathrm{R} \& \mathrm{D})$ was added into additional transwell samples for CXCR2 neutralisation. RFP\% area and EdU incorporation were imaged and analysed as previously described.

Cell culture on polyacrylamide gels. Polyacrylamide gels were prepared according to a published protocol (Dingal et al, 2015). Briefly, top glass coverslips and bottom glass coverslips were sterilised with boiling ethanol for $10 \mathrm{~min}$. The bottom coverslips were rinsed in distilled water, ethanol, chloroform and silanised with chloroform $+0.1 \%$ triethylamine (Sigma) and $0.1 \%$ allyltrichlorosilane (Sigma) for $30 \mathrm{~min}$ at room temperature. The coverslips were then rinsed in chloroform, ethanol, distilled water, and air-dried for storage in a desiccator. Polyacrylamide solution $(0.3 \mathrm{kPa})$ was prepared with $2.6 \%$ acrylamide (Bio-Rad) and $0.07 \%$ bis-acrylamide (Sigma) whereas $40 \mathrm{kPa}$ solution was prepared with $10 \%$ acrylamide and $0.3 \%$ bis-acrylamide in distilled water. $0.1 \%$ ammonium persulfate (Sigma) and $0.1 \%$ tetramethylethylenediamine (Sigma) were added to the solutions to initiate polymerisation. PA solution was sandwiched in between top and silanised bottom coverslips and incubated at room temperature for $30 \mathrm{~min}$ for polymerisation. Sandwiched PA gels were rinsed with phosphate-buffered solution (PBS) and stored at $4{ }^{\circ} \mathrm{C}$ in distilled water. To prepare the gels for cell culture, the top coverslip was removed and the gels were rinsed with PBS and distilled water. Sulfo-SANPAH $\left(5 \mathrm{mg} \mathrm{ml}^{-1}\right)$ (Thermo Fisher) was prepared in $50 \mathrm{~mm}$ HEPES pH 8 and pipetted onto the gels. The cross-linker was activated with $365 \mathrm{~nm}$ ultra-violet light for $10 \mathrm{~min}$ twice. The activated PA gels were rinsed with PBS and distilled water and coated with $30 \mu \mathrm{g} \mathrm{ml}^{-1}$ laminin (Corning) for overnight at $4{ }^{\circ} \mathrm{C}$. The coated gels were rinsed twice with sterile PBS and sterilised with UV light in the culture hood for at least $2 \mathrm{~h}$. LX-2 cells were cultured on the gels in complete medium and immunostained for $\alpha$-SMA, F-actin and Lamin A/C level. Cell area was quantified based on F-actin staining using Image $\mathrm{J}$ and lamin A/C intensity was quantified with ImageJ using a published protocol (McCloy et al, 2014). To assess the induction of cell proliferation, the cells were seeded in DMEM $+0.2 \%$ dialysed FBS. The conditioned media were harvested after $24 \mathrm{~h}$ and applied to MCF7 and MDA- 
MB231 culture to determine the level of EdU incorporation as previously described.

Protein array. LX-1, LX-2, MCF7 and MDA-MB231 cells were seeded and cultured for overnight. The cells were washed and changed the media to HMM. After $24 \mathrm{~h}$, the supernatants were collected and the number of cells was counted. LX-2 cells on PA gels were transferred to new wells containing DMEM $+0.2 \%$ dialysed FBS and supernatants were collected after 6-8h. Protein array staining and analyses were performed according to the manufacturer's protocol (RayBiotech Human Cytokine Array C5). The intensity of selected spots was quantified using ImageJ.

Ex vivo human 3D Liver MPS (LiverChip). 3D liver MPS was assembled according to the manufacturer's recommendation $(\mathrm{CN}$ Bio Innovations Ltd, Oxford, UK). $6 \times 10^{5}$ cells of primary human hepatocytes were seeded in each scaffold in William's $E$ medium (Gibco) supplemented with the Hepatocytes Thawing and Plating Supplement Pack (Life Technologies). The medium was changed to in-house physiologic medium on the next day and after every $48 \mathrm{~h}$. The supernatants were collected and stored at $-80^{\circ} \mathrm{C}$ for downstream assays. $1 \times 10^{3}$ RFP-labelled MDA-MB231 cancer cells were introduced into the MPS on day 3 and treated with $1 \mu \mathrm{m}$ doxorubicin for $72 \mathrm{~h}$ on day 7 . The resulting dormant cancer cells were then stimulated with $20 \mathrm{~nm}$ mouse epidermal growth factor (EGF) (Sigma) and $1 \mu \mathrm{g} \mathrm{ml}^{-1}$ lipopolysaccharides (Sigma) as the positive control or $250 \mathrm{ng} \mathrm{ml}^{-1}$ of IL-8 (R\&D Systems). EdU $(10 \mu \mathrm{M})$ (Life Technologies) was added to the physiologic medium for $96 \mathrm{~h}$. Finally, the scaffolds were fixed with $2 \%$ paraformaldehyde and stained for EdU according to the manufacturer's protocol. RFP\% area for the whole scaffolds were imaged with wide-field $\times 2$ objective lens and confocal microscopy was performed to image for EdU incorporation. For Luminex assay, isolated human NPC fraction was further purified using 25\%:50\% Percoll-gradient centrifugation method. Human primary hepatocytes were cultured \pm equal number of primary NPC in supplemented William's E medium. Media were changed to fresh William's $\mathrm{E}$ medium on day 3 and every $48 \mathrm{~h}$ thereafter. Supernatants were collected for Luminex analyses. The experimental workflow (Supplementary Figure 1) and complete materials and methods are available in the supplementary information.

Statistical analysis. Two-tailed, unequal variance student's $t$-test was used to determine statistical significance between two groups. Kruskal-Wallis one-way ANOVA and multiple comparison Dunn's tests were used to compare the mean for three or more groups. Resulting $P$-values were denoted as follows: $<0.05^{\star}$, $<0.01^{* *},<0.001^{* * *}$. Graphs and statistical analyses were generated using GraphPad Prism (La Jolla, CA, USA).

\section{RESULTS}

Activated stellate cells promote growth of breast cancer cells but not normal breast cells. Previously, our lab showed that coculture with primary human liver NPC promoted breast cancer cells outgrowth (Taylor et al, 2014). Here, we investigated whether the HSCs, another component of the NPC, could promote breast cancer growth independently. We found that MCF7 and MDAMB231 growth were impeded in the minimal HMM, medium used for liver cultures, but the growth of breast cancer cell increased by 3-6-fold when co-cultured with LX-1 cells (Figure 1A). The cocultured samples also showed 1.5-3-fold higher percentages of EdU-positive cells when compared to HMM negative control (Figure 1B). This growth induction, however, was not observed in normal HMEC-1 breast cells, as the cells did not grow and proliferate in LX-1 co-culture (Figure 1A and B). The HSCs were not profoundly affected in the serum-free culture condition as some of the cells were still undergoing cell cycling as indicated by non-RFP EdU-positive cells after 4 days of culture (Supplementary Figure 2).

Some of the proliferating cancer cells in the LX-1 co-culture samples appeared to be contact-independent (white arrows in Supplementary Figure 2) suggesting the involvement of soluble factors. Using a transwell assay, we verified that LX-1-derived soluble factors could indeed promote the growth of MCF7 and MDA-MB231 by 3.8 -fold and 1.9 -fold when compared to the negative controls (Figure 1C). LX-1 soluble factors also imparted significant proliferation advantage to the cancer cells with 1.7 - and 2.8 -fold increase in the percentage of EdU positive in MCF7 and MDA-MB231 cells (Figure 1D).

Activated stellate cells induced higher proliferative fraction than non-activated stellate cells. HSCs cultured on stiff surfaces are in the activated state, marked by the expression of $\alpha$-SMA fibres and pro-collagen 1 and HSP47 gene expression (Supplementary Figures 3 and 4). HSCs cultured on soft gels $(0.3 \mathrm{kPa})$ have been reported to revert back to a less or non-activated state (Caliari et al, 2016). We queried whether cell activation affects tumour cells outgrowth. We utilised LX-2 cells as LX-1 cells adhere poorly to the gels in this media (data not shown). LX-2 cells were derived from an LX-1 clone that can stably grow in low serum medium (Xu et al, 2005). In co-culture setting, LX-2 imparted significant growth and proliferative advantage to the cancer cells only, but not to the normal HMEC- 1 cells, in DMEM $+0.2 \%$ dialysed FBS (Figure $2 \mathrm{~A}$ and B) similar to the effects observed in LX-1 co-culture. LX-2 cells cultured on $0.3 \mathrm{kPa}$ PA gels showed reduced lamin A/C expression indicating that the cells were mechanosensitive to the surface stiffness and LX-2 reversion to a less-activated state was verified by reduced cell area and lacked of and disorganised $\alpha$-SMA fibres when compared to $40 \mathrm{kPa}$ gels culture (Figure 2C and D).

When conditioned medium (CM) from LX-2 cells cultured on $0.3 \mathrm{kPa}$ and $40 \mathrm{kPa}$ gels were used to culture MCF7 and MDAMB231 cells, lower EdU incorporation percentage was observed in cells cultured in $0.3 \mathrm{kPa}-\mathrm{CM}$ for both MCF7 and MDAMB231 cells (Figure 2E). Thus, our results indicated that activated HSCs secrete soluble factors to induce higher cancer proliferation.

Non-parenchymal cells and hepatic stellate cells secreted high level of IL-8 and MCP-1. Next, we utilised protein array and Luminex assays to determine the chemokines/growth factors secreted by the activated HSCs and primary human NPC. Protein array analyses revealed high level of IL-8, IL-6, GRO- $\beta \gamma$ and MCP1 secretion by the LX-1 cells in vitro. MDA-MB231 cells secreted GROs and IL-8 but at lower level when normalised to the loading positive controls and cell number (Figure 3A and B). (The map for the whole array is available in the Supplementary Figure 5.) Less activated LX-2 cells, cultured on $0.3 \mathrm{kPa}$ PA gels, secreted lower levels of these pro-inflammatory cytokines especially IL-8 (Figure 3C). Interestingly, primary human NPC, cultured on stiff scaffolds in 3D liver MPS, secreted higher level of IL-8, MCP-1, IL6 and IP-10 when compared to primary hepatocytes alone (Figure 3D). Based on these results, we postulated that inflammatory chemokines, namely IL-8 and MCP-1, could be responsible for HSC-induced cancer growth.

IL-8 but not MCP-1 directly promoted MDA-MB231 survival in vitro. Next, we tested whether these chemokines could directly affect cancer cell growth in vitro. In serum-free medium (SFM), MCF7 cells did not respond to IL-8 although the EGF-treated positive control showed significant growth induction (Figure 4A).

In MDA-MB231 cells, EGF treatment did not promote cell growth but significantly alleviated serum-deprived growth arrest (Figure 4B). Likewise, the addition of IL-8 in SFM significantly 

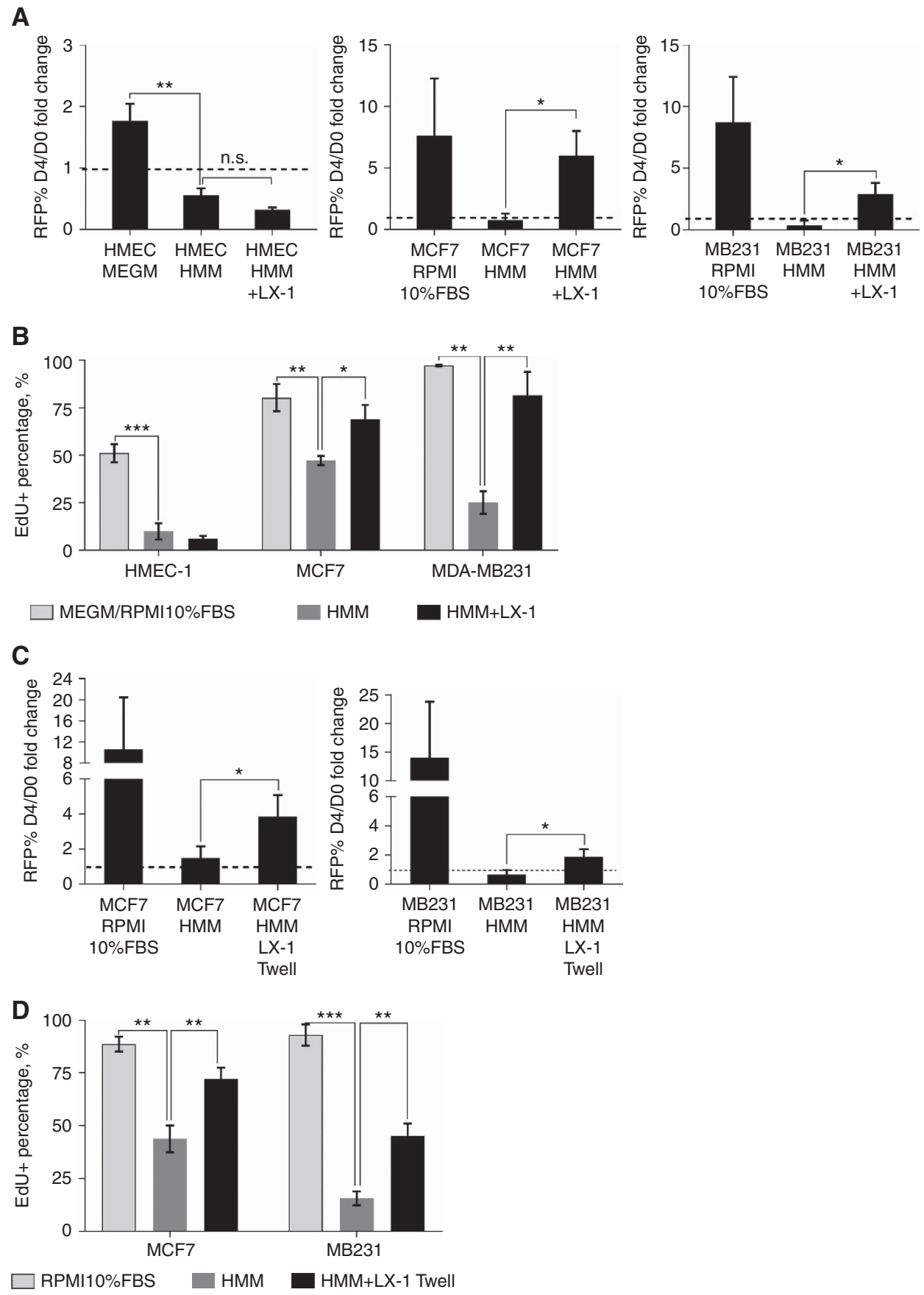

Figure 1. LX-1 promotes breast cancer cell growth and proliferation in vitro. Serum-free HMM and complete media (RPMI 10\%FBS or MEGM) serve as the negative and positive controls, respectively. Average RFP\% area fold-change (A) and average EdU incorporation percentage (B) with standard deviation (SD) of normal breast HMEC-1, MCF7 and MDA-MB231 cells in LX-1 co-culture $(n=3)$. Average RFP\% area fold change (C) and average EdU incorporation percentage (D) with SD of MCF7 and MDA-MB231 in LX-1 transwell separate culture. $n=4$ for MCF7 and $n=3$ for MDA-MB231. ${ }^{\star} P<0.05,{ }^{*} * P<0.01,{ }^{\star \star \star} P<<0.001$.

sustained MDA-MB231 cell when compared to the negative control (Figure 4B). MCP-1 did not affect the growth of both MCF7 and MDA-MB231 (Figure 4A and B), suggesting that its effects, if any, would be indirect to the cancer cells.

To verify the effects of IL-8 in promoting cancer cell growth and survival in vitro, cancer cells cultured with LX-1-transwell insert were treated with CXCR2 neutralising antibody. Similar with our previous results, LX-1 transwell insert augmented cancer growth by 2-3 folds (Figure 4C and D). Blocking CXCR2 did not affect MCF7 growth and proliferation (Figure 4C) but significantly decreased LX-1-induced MDA-MB231 cancer growth and proliferation (Figure 4D).
IL-8 stimulated dormant MDA-MB231 growth in 3D liver MPS. Next, we examined the effects of IL-8 on dormant tumour cells in the 3D liver MPS, a near physiological situation that can determine the effects of isolated stimuli (Wheeler et al, 2014). LPS + EGF treatment, serving as the positive control, induced dormant cells to significantly grow and proliferate (Figure $5 \mathrm{~B}$ and C). Addition of $0.25 \mu \mathrm{g} \mathrm{ml}^{-1}$ of IL- 8 into the physiologic medium also induced tumour cell escape from dormancy as indicated by two-fold increase in RFP\% area and promoted EdU incorporation in about $50 \%$ of the cells, similar to the level seen in the positive control (Figure 5B and C). There were also some EdU-positive cells in the doxorubicin-only negative control but most of these cells 
A
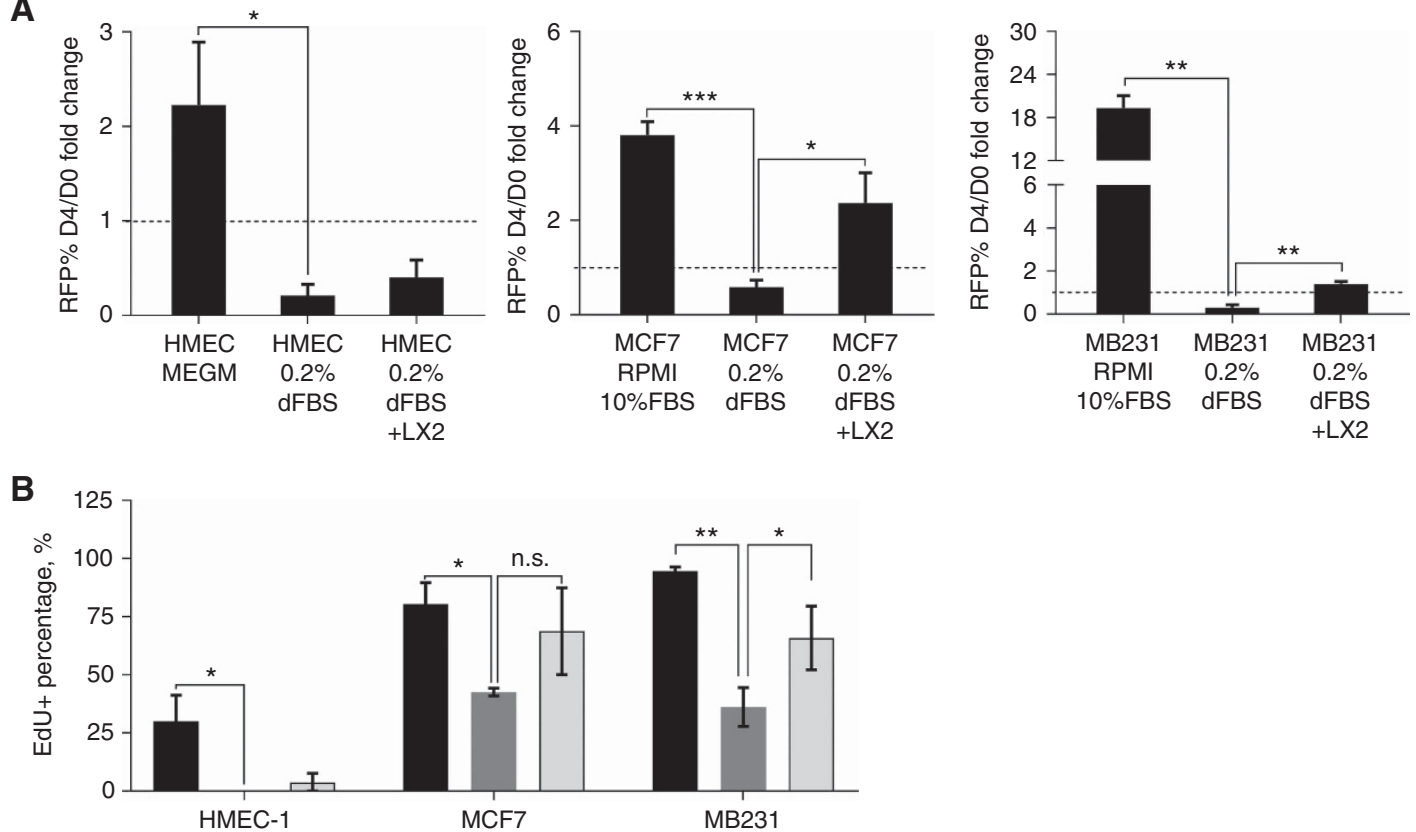

MEGM/RPMI10\%FBS

$0.2 \% \mathrm{dFBS}$

$0.2 \% \mathrm{dFBS}+\mathrm{LX} 2$

C
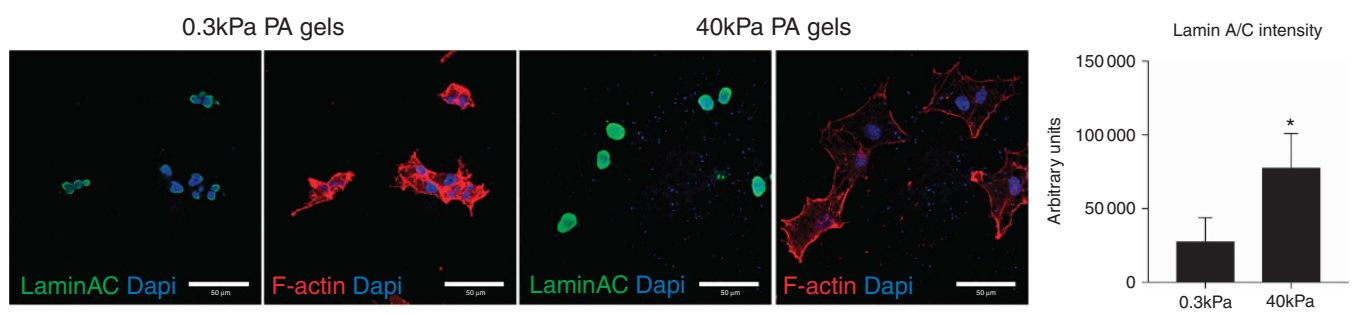

D
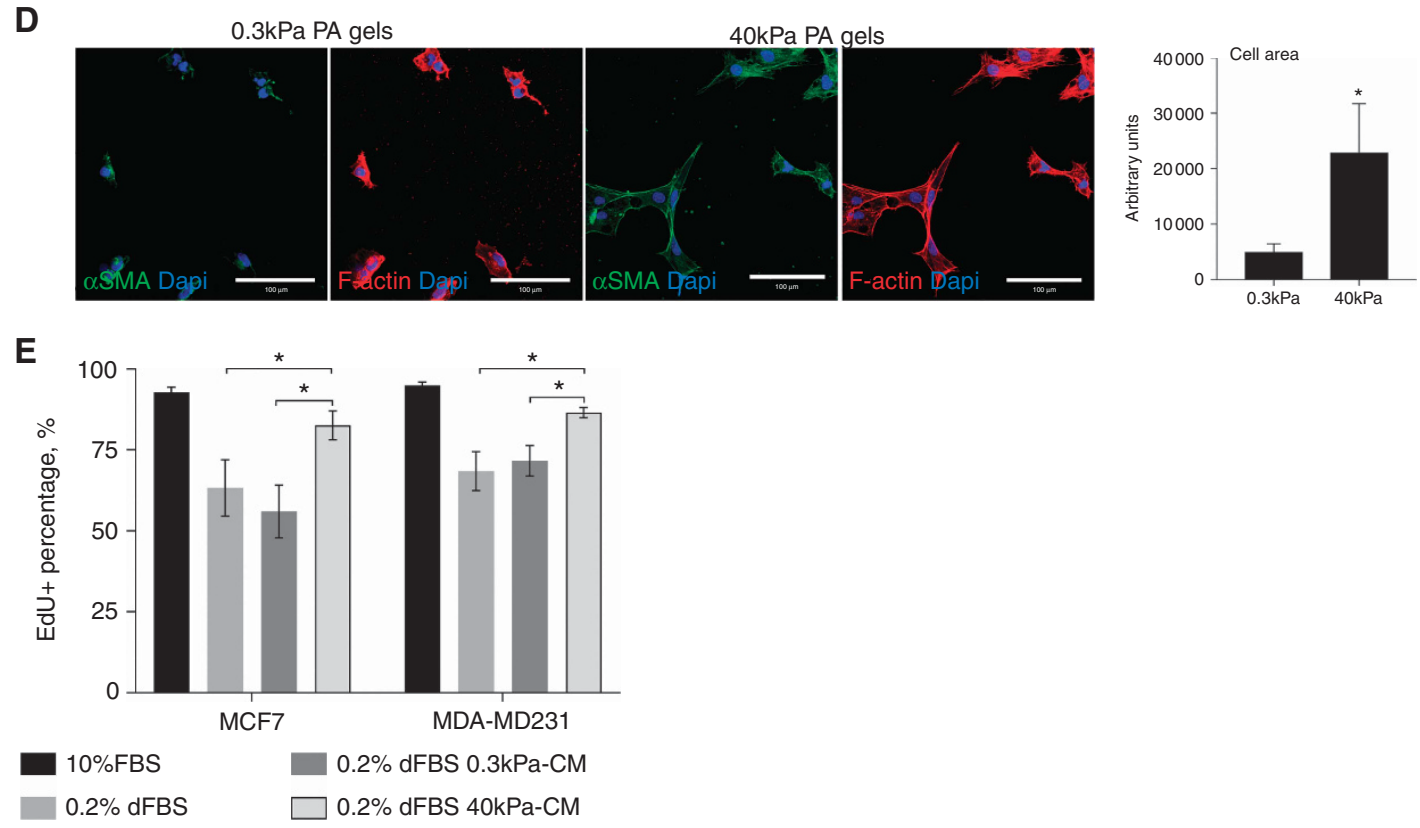

Figure 2. Activated stellate cells trigger higher proliferative induction than less-activated stellate cells. DMEM $+0.2 \%$ dialysed FBS and $\mathrm{RPMI}+10 \% \mathrm{FBS}$ serve as the negative and positive controls, respectively. Average RFP\% area fold change (A) and average EdU incorporation percentage (B) with SD of HMEC-1, MCF7 and MDA-MB231 cells in LX-2 co-culture $(n=3)$. (C) Representative images of LX-2 lamin A/C immunofluorescence and average lamin A/C intensity with SD $(n=4)$. Scale bar $=50 \mu \mathrm{m}$. (D) Representative images of LX-2 $\alpha \mathrm{SMA}$ immunofluorescence and average cell area with SD $(n=3)$. Scale bar $=100 \mu \mathrm{m}$. (E) Average EdU incorporation percentage in MCF7 and MDAMB231 cultured with $100 \%$ conditioned-media (CM) from $0.3 \mathrm{kPa}$ and $40 \mathrm{kPa} \mathrm{LX}-2$ cultures for $48 \mathrm{~h}(n=3)$. ${ }^{\star} P<0.05,{ }^{\star \star} P<0.01,{ }^{\star \star \star} P<0.001$. 
A

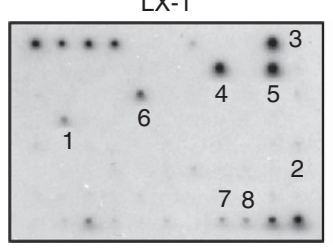

MCF-7

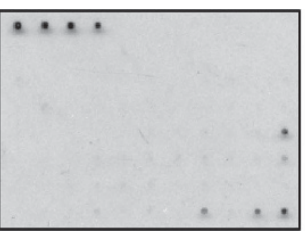

MDA-MB231

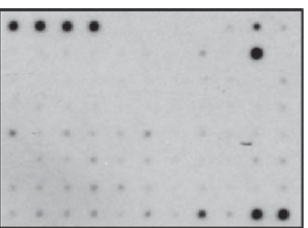

B

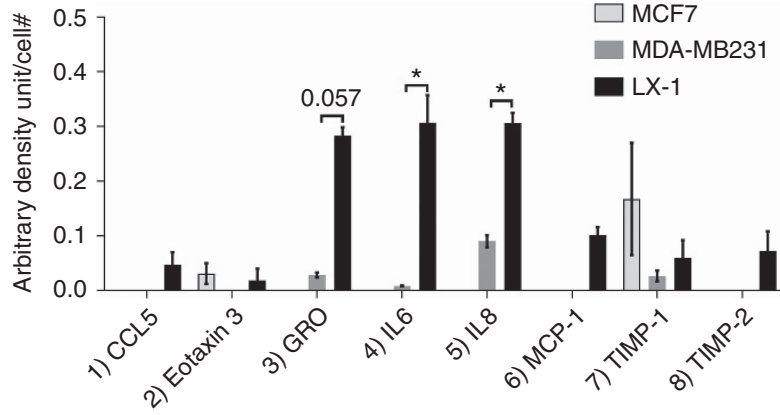

C

$0.3 \mathrm{kPa}$

$40 \mathrm{kPa}$
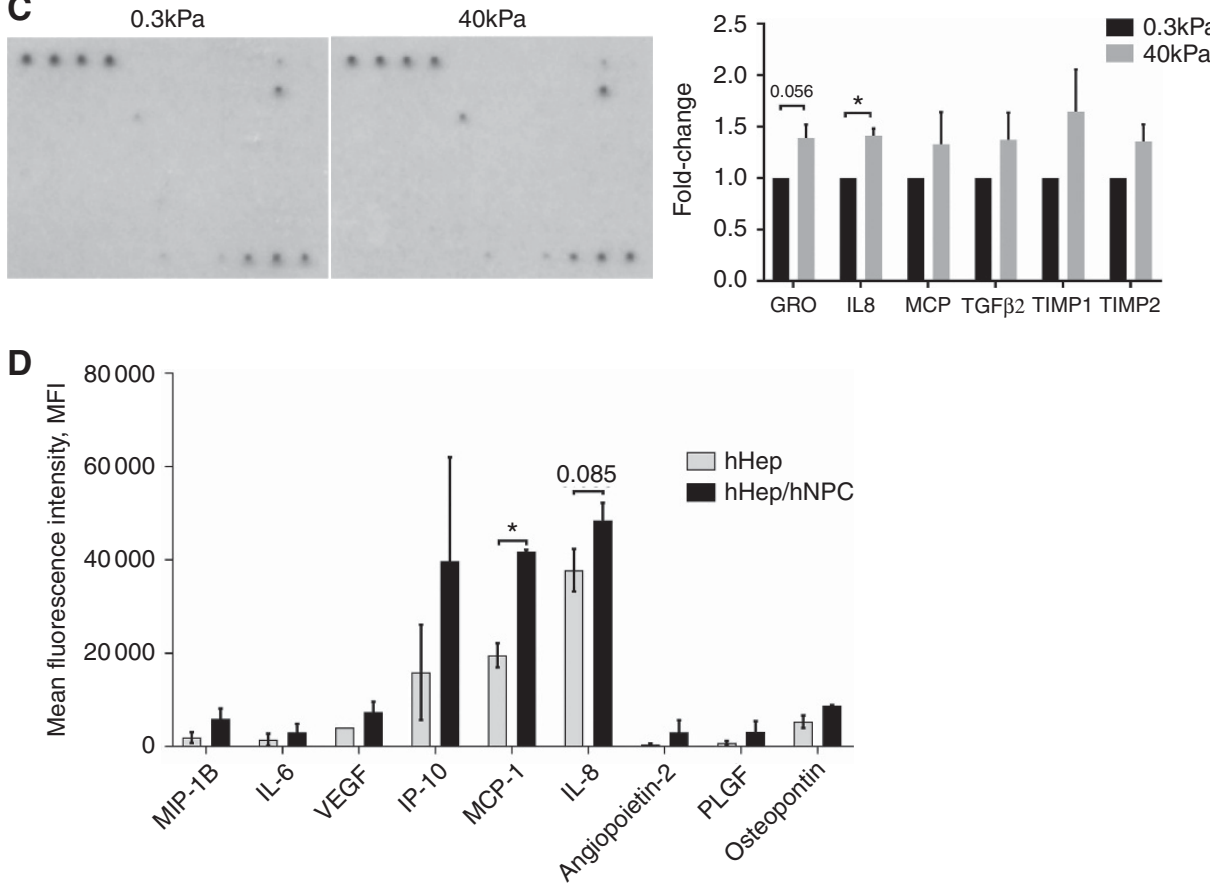

Figure 3. Activated HSCs and primary liver NPCs secrete high level of IL-8 and MCP-1. (A) Representative images of protein array analysis on cells cultured in HMM and (B) average density of selected cytokines corrected to reference positive controls and normalised to cell number with SD $(n=2)$. (C) Representative images of secretome analysis of LX-2 cells cultured on $0.3 \mathrm{kPa}$ and $40 \mathrm{kPa}$ PA gels in DMEM $+0.2 \%$ dialyzed FBS and average spot density of selected cytokines normalised to cell number with SD $(n=4)$. (D) Average mean fluorescence intensity (MFI) of chemokines/cytokines with standard error of the mean measured using Luminex assay on supernatants from primary human hepatocytes \pm NPC cultured in 3D Liver MPS in serum-free William's E medium ( $n=2$ donors). ${ }^{\star} P<0.05$.

were on the stiff polystyrene bridges between the pores of the scaffold, an area that does not allow for dormancy as previously observed (Clark et al, 2017). IL-8 induced cancer cell proliferation even within the soft liver tissue (green arrow heads in Figure 5A, right panel). Prolonged cell culture and treatments with cytokine, growth factors, low dose doxorubicin or LPS did not adversely affect the health and functions of the hepatic tissues as the injury markers, AST and ALT, remained at low levels throughout the whole experiment and the cells maintained blood urea nitrogen production and CYP enzymes metabolic activities (Supplementary Figure 6).

IL-8 induced ERK phosphorylation in MDA-MB231 cells to alleviate growth suppression. Previously, it was reported that a high ERK/P38 ratio indicated tumour outgrowth whereas low ERK/P38 ratio corresponded to growth arrest in several tumour cell lines (Aguirre-Ghiso et al, 2003). We verified that, in MDAMB231 cells, IL-8 treatment significantly increased the level of phosphorylated ERK when compared to the negative control, SFM, and the addition of SCH772984, an ERK-specific inhibitor, blocked IL-8 induced ERK phosphorylation (Figure 6B, middle and right panels). Augmented ERK phosphorylation correlated with a significant increase in cancer cell survival when IL-8 was added into SFM which was reversed with SCH772984 treatment (Figure 6B, left panel). Chemokine and inhibitor treatments did not affect ERK phosphorylation and cancer growth in MCF7 (Figure 6A). This suggests that inherent E-cadherin signalling in the MCF7 confers much of the ERK signalling (Ma et al, 2016). 

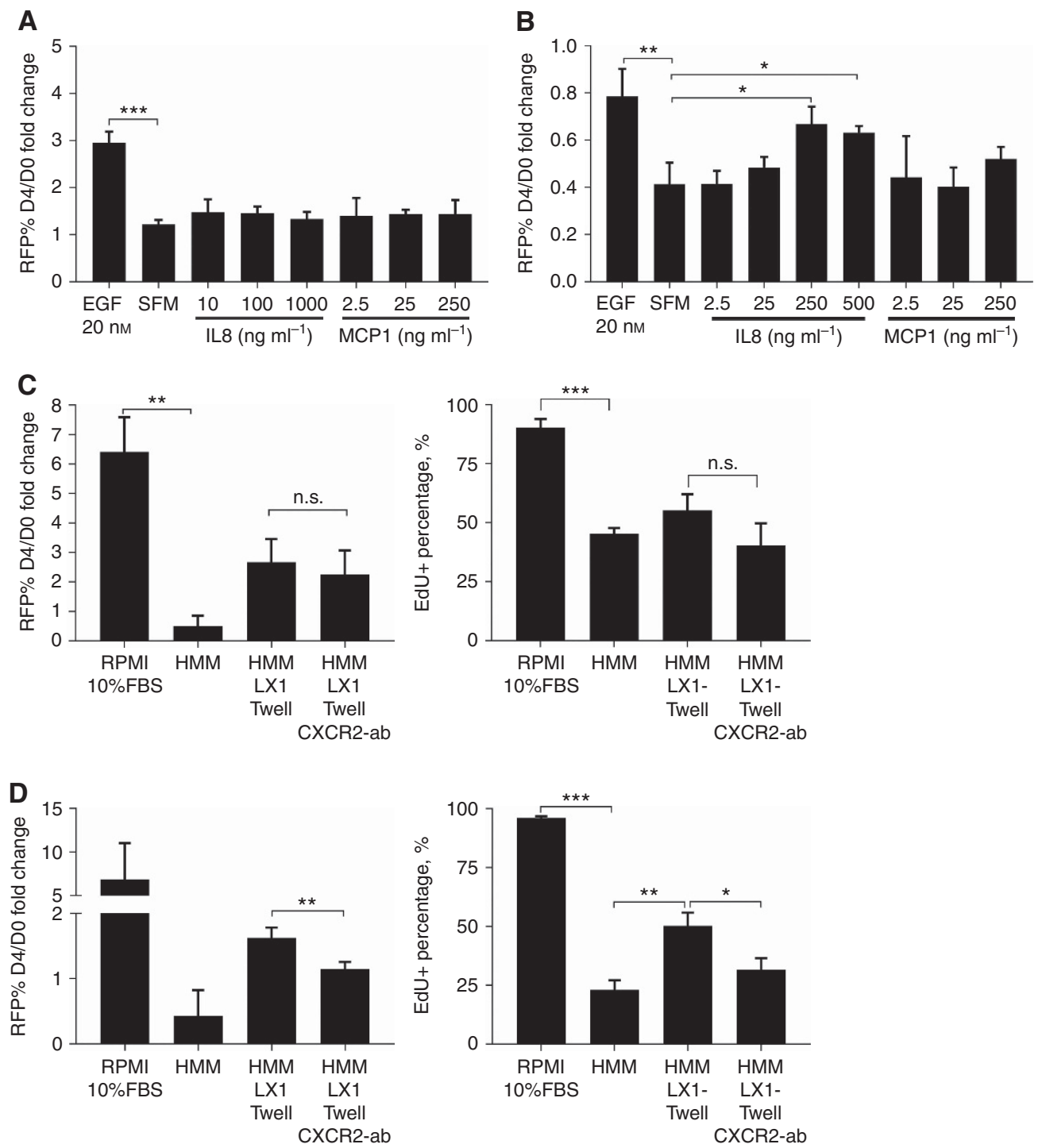

Figure 4. IL-8, but not MCP-1, alleviated serum starvation-induced growth arrest in MDA-MB231 cells in vitro. Serum-free medium (SFM) and $\mathrm{SFM}+20 \mathrm{~nm}$ epidermal growth factor (EGF) serve as the negative and positive controls, respectively. Average RFP\% growth fold-change with SD of MCF7 (A) and MDA-MB231 cells (B) in SFM with chemokine treatment at the indicated concentration $(n=4)$. Average RFP\% fold-change and average EdU incorporation percentage with SD of MCF7 cells (C) and MDA-MB231 (D) cultured with LX-1-transwell insert $\pm 1 \mu \mathrm{g} \mathrm{ml}{ }^{-1}$ CXCR2 antibody $(n=3) .{ }^{\star} P<0.05,{ }^{\star *} P<0.01,{ }^{\star \star *} P<0.001$.

(The immunoblot results for all of the experimental replicates are available in Supplementary Figure 7.)

\section{DISCUSSION}

The factors that drive cancer to re-emerge after long latency periods are still unclear. We hypothesised that liver inflammation stimulates or activates the liver NPCs to secrete pro-inflammatory signals to drive metastatic outgrowth. Here, we found that activated human stellate cells could indeed drive breast cancer cell growth and proliferation by secreting various tumourpromoting soluble factors and we verified that IL-8, one of the most highly secreted cytokines, can directly promote tumour growth in vitro and ex vivo.

IL-8 is a well-known chemokine that mediates cell motility, invasion and metastasis by inducing cancer cells to undergo EMT (Fernando et al, 2011). However, the role of IL-8 in cancer cell proliferation remains controversial. Several studies reported that IL-
8 could not induce cell proliferation in vitro (Freund et al, 2003; Yao et al, 2007) and repressed in vivo cancer growth by promoting neutrophil recruitment (Hirose et al, 1995; Lee et al, 2000). However, it was also reported that IL-8 increased cyclin D1 expression as well as $\mathrm{PI} 3 \mathrm{~K} / \mathrm{Akt} / \mathrm{mTOR}$ activation in DU145 and PC3 prostate cancer cells, and blocking IL-8 expression abrogated prostate cancer cell proliferation (MacManus et al, 2007). Moreover, IL-8 significantly promoted the proliferation of NSCLC (Luppi et al, 2007) and colon cancer cell (Lee et al, 2012) in vitro and in vivo. As we found that IL8 is significantly increased by stressed HSCs and stimulated liver NPCs, we queried whether IL-8 could sufficiently induce cell proliferation to facilitate tumour escape from dormancy. In line with previously published results, we found that IL-8 treatment did not confer significant growth advantage to the cancer cells but could merely maintain MDA-MB231 cell survival in serum-free condition in vitro. However, IL-8 imparted a significant increment in cancer cell growth and proliferation in the 3D liver MPS, in a microenvironment that is richer in signals than the $2 \mathrm{D}$ co-culture. Blocking CXCR2, a receptor for both IL-8 and GRO, substantially reduced cytokine-induced proliferation. 
A
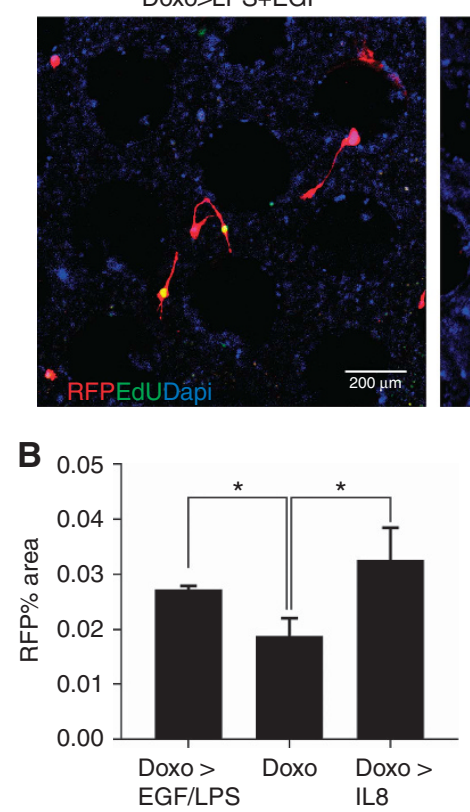

Doxo

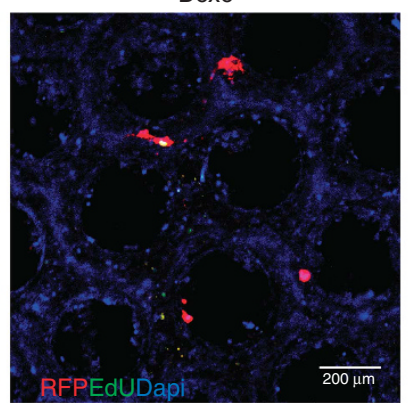

Doxo>IL-8

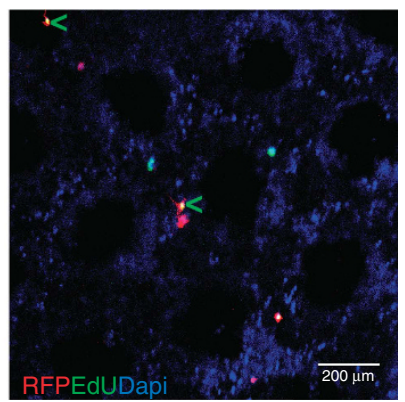

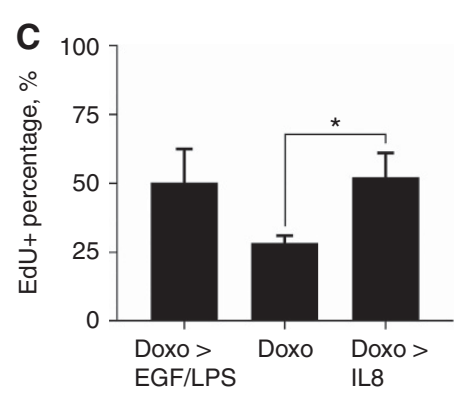

Figure 5. IL-8 drives cancer cell emergence from dormancy in 3D Liver MPS. (A) Representative images of scaffolds with doxorubicin followed by LPS + EGF treatments (positive control), doxorubicin only (negative control) and doxorubicin followed by $0.25 \mu \mathrm{g} \mathrm{ml}^{-1} \mathrm{IL-}-8 \mathrm{treatment}$. Green arrow heads point to proliferating RFP-labelled cancer cells in the soft liver tissues. Scale bar $=200 \mu \mathrm{m}$. Average RFP\% area (B) and average EdU positive percentage (C) with SD $(n=3)$. ${ }^{\star} P<0.05$.

A
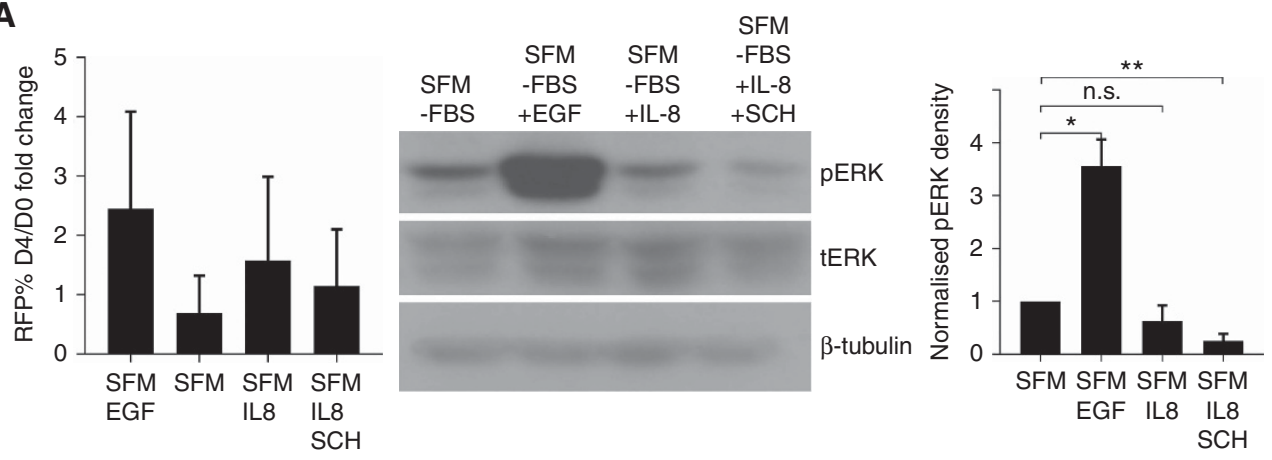

B
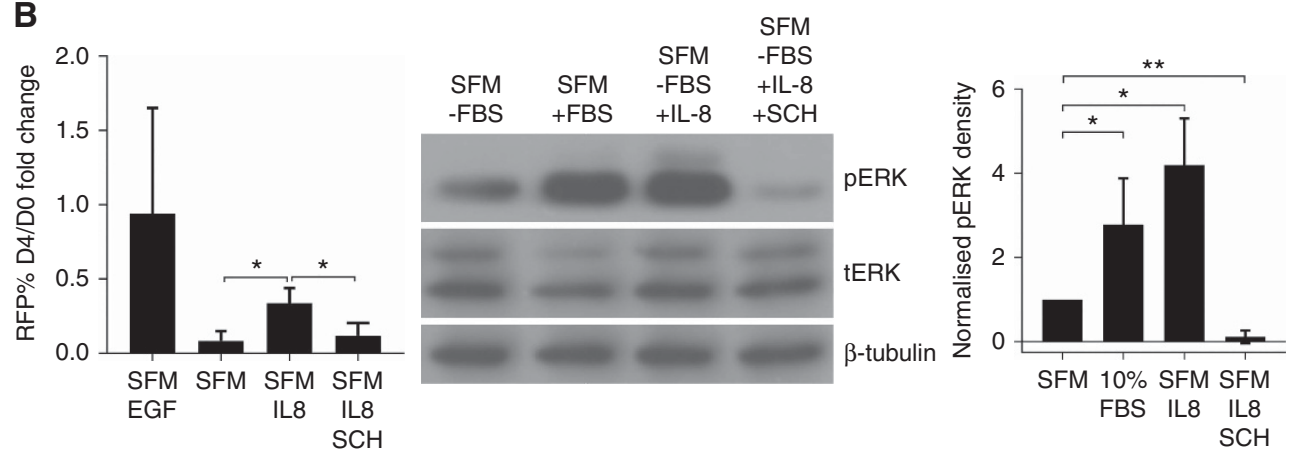

Figure 6. IL-8 induces ERK phosphorylation. Serum-free RPMI medium (SFM) and SFM + EGF/RPMI + 10\% FBS serve as the negative and positive controls, respectively. Average RFP\% growth fold-change (left panel), immunoblot for phospho-ERK (pERK), total ERK (tERK) and tubulin (middle panel) and pERK density quantification (right panel) of MCF7 (A) and MDA-MB231 (B) cells following 4 days of IL-8 treatments with SD ( $n=3, n=4$ for MCF7 immunoblot). Treatments: $1 \mu \mathrm{g} \mathrm{ml}^{-1} \mathrm{IL}-8$ for MCF7, $0.25 \mu \mathrm{g} \mathrm{ml}^{-1} \mathrm{IL}-8$ for MDA-MB231, $20 \mathrm{nM}$ EGF and $0.5 \mu \mathrm{M} \mathrm{SCH772984.}{ }^{*} P<0.05$, $\star \star P<0.01$.

These findings are clinically relevant as prognostic analyses using publicly available data reveals shorter metastasis-free survival in breast cancer patients with high IL- 8 gene expression in six out eight patient cohorts (Supplementary Figure 8) (Goswami and Nakshatri, 2013). Additionally, elevated IL-8 serum level correlated significantly with advance disease stage and metastasis (Ahmed et al, 2006; Derin et al, 2007; Ma et al, 2017). Several antibodies targeting IL- 8 or CXCR $1 / 2$ are currently undergoing clinical trials for breast cancer treatments (ClinicalTrials.gov [Internet], 2015a, b). Hence, IL-8/CXCR2 could be a relevant target for metastasis prevention and may be crucial in driving tumour outgrowth from dormancy. Furthermore, these 
findings support the use of the 3D liver MPS in deriving candidate markers or targets.

It is important to note that other chemokines and cytokines also contribute either directly or indirectly to cancer cell growth since neutralisation of CXCR2 did not fully abrogate cancer growth in the transwell assays. These factors could create feedback loops to increase the secretion of other inflammatory cytokines and growth factors by other cells in the liver. MCP-1/CCL2, unlike IL-8, was highly and exclusively secreted by the liver NPCs but did not induce significant cancer growth. It was previously reported that breast cancer cells and tissues expressed high levels of both MCP-1 and its receptor CCR2 which conferred survival, but not proliferative, advantage to the breast cancer cells (Fang et al, 2012). MCP-1 may indirectly affect tumour outgrowth by recruiting inflammatory monocytes and facilitating efficient tumour cell extravasation for metastatic seeding (Qian et al, 2011) or inducing monocyte polarisation into the alternative, protumour M2 phenotype (Roca et al, 2009). Linking this with our finding of M2-like macrophages driving a mesenchymal reversion (Yang et al, 2016) would provide an indirect mechanism for promoting emergence. IL-6 is another pro-inflammatory cytokine secreted by both primary human NPCs and LX-1 cells and has been shown to induce MCF7 growth and spheroid formation (Sansone et al, 2007). Another candidate could be TGF $\beta$, a potent inducer of HSC activation and IL-8 secretion. Interestingly, we observed a slight induction of TGF $\beta 2$ secretion by the LX- 2 cells cultured on the stiff $40 \mathrm{kPa}$ gels when compared to soft $0.3 \mathrm{kPa}$ gels (Figure 3C).

In this study, we strove to closely recapitulate human physiology. However, the isolation of HSCs from primary human NPC fraction proved to be quite challenging due to limited availability of the cells and viability during isolation. We therefore opted to use human HSC lines. The human LX-1 and LX-2 HSC lines were established in the laboratory of Dr Scott Friedman at Icahn School of Medicine at Mount Sinai, NY, USA (Xu et al, 2005). We validated that both cell lines expressed HSC markers such as $\alpha$-SMA and GFAP but not endothelial cell markers CD31 or vWF (Supplementary Figure 3). The cells also secreted TIMP-1 and TIMP-2 as shown in the protein array (Figure 3A and C). Finally, the cells showed typical HSC response to TGF- $\beta 1$ by increasing pro-collagen 1 and HSP47 gene expression (Supplementary Figure 4). The key effect of HSC on cancer cell growth was validated with TWNT-1 and HSC-T6 cell lines where cancer cell growth induction was found to be significantly augmented and was comparable to TMNK-1 endothelial cellinduced outgrowth as published previously (Taylor et al, 2014) (Supplementary Figure 9).

There are several results and experimental settings that require further discussion. First of all, although mesenchymal-like MDAMB231 cells, derived from triple negative breast cancer (TNBC), are the more aggressive cancer cells than the epithelial-like MCF7 cells, our co-culture and transwell assays using HMM showed higher fold-change in MCF7 growth when compared to MDAMB231. We observed that the MDA-MB231 cells are highly dependent on serum supplement as evident by higher uncorrected RFP\% area in the RPMI $+10 \%$ FBS-positive controls (4-7\%) when compared to MCF7-positive controls ( $2 \%)$. The negative effect of serum-free media could be rescued by co-culturing the cancer cells with HSCs or by treating the cancer cells with IL-8. Second, we observed weaker growth in the transwell assays when compared to co-culture experiments. This is likely due to dilutional effects where higher medium volume is required for the transwell assay and HSC growth area in the transwell insert $\left(0.33 \mathrm{~cm}^{2}\right)$ is five times less than 24-well plate $\left(1.88 \mathrm{~cm}^{2}\right)$. Third, less prominent effects were observed in IL- 8 treatment on MCF7. This is due to lower level of CXCR2 expression in MCF7 cells when compared to MDA-MB231 cells. CXCR1 is not expressed by both cell types (our unpublished data) (Freund et al, 2003). Fourth, the cytokine array indicated that MDA-MD231 cells also secreted IL-8. However, the secretion was lower than LX-1 cells when normalised to their respective cell numbers. It must be noted that these studies were performed under conditions wherein the single cancer cells were initially $<1 \%$ of the total cells in the $3 \mathrm{D}$ liver MPS. Similar, in tumour dormancy setting, in which we use doxorubicin to remove cycling cells as the dormant cells are insensitive to the treatment, the cancer cells are relatively scant in number. Therefore, we presume that the surrounding stromal cells were the major contributor of IL-8 or other factors. Lastly, only MDA-MB231 cells were used in the 3D liver MPS to match in vivo and clinical observations where TNBCs show a higher propensity to metastasise to the liver (Yuan et al, 2014) and unlike MCF-7, MDAMB231 cells could spontaneously metastasise to various organs in vivo (Holliday and Speirs, 2011).

HSCs are one of the main components of the liver NPC. The roles and mechanisms for HSC-induced tumour growth in mouse models have been reported (Coulouarn et al, 2012; Zhao et al, 2011). However, since there are various processes and complex interactions between HSCs and their microenvironment, it is difficult to pinpoint the specific effects of HSC activation in driving tumour growth. Thus, in order to directly compare tumour growth induction by quiescent and activated HSCs, we cultured LX-2 cells on $0.3 \mathrm{kPa}$ PA gel to model physiologic liver stiffness and $40 \mathrm{kPa}$ PA gel to model diseased liver. Here, the LX-2 cells reverted to a less activated state in vitro as marked by reduced expression of nuclear lamin A/C and lack of $\alpha$-SMA fibres on soft PA gels, similar with published observation (Swift et al, 2013; Caliari et al, 2016) and resulted in reduced cancer proliferation induction. The percentages of EdU incorporation for these experiments were higher than other results due to shorter experimental period but a significant difference in proliferation induction was still observed. The fold-change of cytokine secretion in cells cultured on stiff gels, though marginal, is replicable and statistically significant. Similar results were also reported where pro-inflammatory cytokines were slightly upregulated in activated primary rat HSCs when compared to quiescent HSCs (Jiang et al, 2006). Persistence of upregulation of pro-inflammatory cytokines may exert more profound effects in patients over months as the dormant cancers cells are constantly being exposed to elevated chemokines level for a longer period instead of just for 4-6 experimental days.

In summary, we showed here that human HSCs and their derived IL- 8 could induce breast cancer growth and proliferation. As this factor is expressed upon stressors activating the HSCs, this could link systemic inflammation to breast cancer emergence and outgrowth. Furthermore, we found that IL-8 prolonged in vitro cell survival under serum-starved condition and potentiated cancer growth and proliferation in the 3D liver MPS tumour dormancy model through ERK activation. This aspect of survival could also explain the generalised chemoresistance of metastases. This study suggests that preventing liver inflammation or specifically inhibiting key inflammation inducers might be beneficial to prevent delayed tumour recurrence or to re-establish chemoresponsiveness.

\section{ACKNOWLEDGEMENTS}

We would like to thank the Wells lab members for their insightful comments and suggestions. We also would like to thank Dr Scott Friedman and Dr Alex Soto-Gutierrez for providing the cell lines used in this study, Dr Carissa Young for Luminex analysis and Dr Raman Venkataramanan for CYP substrate analysis. This study was supported by grants from the NIH UH3 TR000496 and VA Merit Award. The imaging was done using microscopes 
funded by the NIH (Grant numbers: 1S10RR028478-01 and 1S10OD019973-01).

\section{CONFLICT OF INTEREST}

A Wells holds a patent position on the 3D MPS LiverChip now being commercialised by $\mathrm{CN}$ Bio Innovations Ltd. All other authors declare no conflict of interest.

\section{REFERENCES}

Aguirre-Ghiso JA, Estrada Y, Liu D, Ossowski L (2003) ERK(MAPK) activity as a determinant of tumor growth and dormancy; regulation by p38(SAPK). Cancer Res 63(7): 1684-1695.

Ahmed OI, Adel AM, Diab DR, Gobran NS (2006) Prognostic value of serum level of interleukin- 6 and interleukin- 8 in metastatic breast cancer patients. Egypt J Immunol 13(2): 61-68.

Caliari SR, Perepelyuk M, Soulas EM, Lee GY, Wells RG, Burdick JA (2016) Gradually softening hydrogels for modeling hepatic stellate cell behavior during fibrosis regression. Integr Biol (Camb) 8(6): 720-728.

Chao Y, Wu Q, Shepard C, Wells A (2012) Hepatocyte induced re-expression of E-cadherin in breast and prostate cancer cells increases chemoresistance. Clin Exp Metastasis 29(1): 39-50.

Chao YL, Shepard CR, Wells A (2010) Breast carcinoma cells re-express E-cadherin during mesenchymal to epithelial reverting transition. Mol Cancer 9(1): 179.

Clark AM, Wheeler SE, Young CL, Stockdale L, Shepard Neiman J, Zhao W, Stolz DB, Venkataramanan R, Lauffenburger D, Griffith L, Wells A (2017) A liver microphysiological system of tumor cell dormancy and inflammatory responsiveness is affected by scaffold properties. Lab Chip 17(1): 156-168.

ClinicalTrials.gov [Internet] (2015a) A Double-blind Study of Paclitaxel in Combination with Reparixin or Placebo for Metastatic Triple-Negative Breast Cancer (FRIDA). National Library of Medicine (US): Bethesda, MD.

ClinicalTrials.gov [Internet] (2015b) HuMax-IL8 (Interleukin8) in Patients with Advanced Malignant Solid Tumors. National Library of Medicine (US): Bethesda, MD.

Coulouarn C, Corlu A, Glaise D, Guenon I, Thorgeirsson SS, Clement B (2012) Hepatocyte-stellate cell cross-talk in the liver engenders a permissive inflammatory microenvironment that drives progression in hepatocellular carcinoma. Cancer Res 72(10): 2533-2542.

Derin D, Soydinc HO, Guney N, Tas F, Camlica H, Duranyildiz D, Yasasever V, Topuz E (2007) Serum IL-8 and IL-12 levels in breast cancer Med Oncol 24(2): 163-168.

Dingal PC, Bradshaw AM, Cho S, Raab M, Buxboim A, Swift J, Discher DE (2015) Fractal heterogeneity in minimal matrix models of scars modulates stiff-niche stem-cell responses via nuclear exit of a mechanorepressor. Nat Mater 14(9): 951-960.

Fang WB, Jokar I, Zou A, Lambert D, Dendukuri P, Cheng N (2012) CCL2/ CCR2 chemokine signaling coordinates survival and motility of breast cancer cells through Smad3 protein- and p42/44 mitogen-activated protein kinase (MAPK)-dependent mechanisms. J Biol Chem 287(43): 36593-36608.

Fernando RI, Castillo MD, Litzinger M, Hamilton DH, Palena C (2011) IL-8 signaling plays a critical role in the epithelial-mesenchymal transition of human carcinoma cells. Cancer Res 71(15): 5296-5306.

Freund A, Chauveau C, Brouillet JP, Lucas A, Lacroix M, Licznar A, Vignon F, Lazennec G (2003) IL-8 expression and its possible relationship with estrogen-receptor-negative status of breast cancer cells. Oncogene 22(2): 256-265.

Friedman SL (2008) Hepatic stellate cells: protean, multifunctional, and enigmatic cells of the liver. Physiol Rev 88(1): 125-172.

Fujita T, Soontrapa K, Ito Y, Iwaisako K, Moniaga CS, Asagiri M, Majima M, Narumiya S (2016) Hepatic stellate cells relay inflammation signaling from sinusoids to parenchyma in mouse models of immune-mediated hepatitis. Hepatology 63(4): 1325-1339.

Ghajar CM, Peinado H, Mori H, Matei IR, Evason KJ, Brazier H, Almeida D, Koller A, Hajjar KA, Stainier DY, Chen EI, Lyden D, Bissell MJ (2013) The perivascular niche regulates breast tumour dormancy. Nat Cell Biol 15(7): 807-817.
Goswami CP, Nakshatri H (2013) PROGgene: gene expression based survival analysis web application for multiple cancers. J Clin Bioinform 3(1): 22.

Hirose K, Hakozaki M, Nyunoya Y, Kobayashi Y, Matsushita K, Takenouchi T, Mikata A, Mukaida N, Matsushima K (1995) Chemokine gene transfection into tumour cells reduced tumorigenicity in nude mice in association with neutrophilic infiltration. Br J Cancer 72(3): 708-714.

Holliday DL, Speirs V (2011) Choosing the right cell line for breast cancer research. Breast Cancer Res 13(4): 215.

Jiang F, Parsons CJ, Stefanovic B (2006) Gene expression profile of quiescent and activated rat hepatic stellate cells implicates Wnt signaling pathway in activation. J Hepatol 45(3): 401-409.

Karrison TG, Ferguson DJ, Meier P (1999) Dormancy of mammary carcinoma after mastectomy. J Natl Cancer Inst 91(1): 80-85.

Lee LF, Hellendall RP, Wang Y, Haskill JS, Mukaida N, Matsushima K, Ting JP (2000) IL-8 reduced tumorigenicity of human ovarian cancer in vivo due to neutrophil infiltration. J Immunol 164(5): 2769-2775.

Lee Y-TM (1985) Patterns of metastasis and natural courses of breast carcinoma. Cancer Metastasis Rev 4: 153-172.

Lee YS, Choi I, Ning Y, Kim NY, Khatchadourian V, Yang D, Chung HK, Choi D, LaBonte MJ, Ladner RD, Nagulapalli Venkata KC, Rosenberg DO, Petasis NA, Lenz HJ, Hong YK (2012) Interleukin-8 and its receptor CXCR2 in the tumour microenvironment promote colon cancer growth, progression and metastasis. Br J Cancer 106(11): 1833-1841.

Luppi F, Longo AM, de Boer WI, Rabe KF, Hiemstra PS (2007) Interleukin-8 stimulates cell proliferation in non-small cell lung cancer through epidermal growth factor receptor transactivation. Lung Cancer 56(1): 25-33.

Ma B, Wheeler SE, Clark AM, Whaley DL, Yang M, Wells A (2016) Liver protects metastatic prostate cancer from induced death by activating E-cadherin signaling. Hepatology 64(5): 1725-1742.

Ma Y, Ren Y, Dai ZJ, Wu CJ, Ji YH, Xu J (2017) IL-6, IL-8 and TNF-alpha levels correlate with disease stage in breast cancer patients. Adv Clin Exp Med 26(3): 421-426.

MacManus CF, Pettigrew J, Seaton A, Wilson C, Maxwell PJ, Berlingeri S, Purcell C, McGurk M, Johnston PG, Waugh DJ (2007) Interleukin-8 signaling promotes translational regulation of cyclin D in androgenindependent prostate cancer cells. Mol Cancer Res 5(7): 737-748.

Magnus N, Garnier D, Meehan B, McGraw S, Lee TH, Caron M, Bourque G, Milsom C, Jabado N, Trasler J, Pawlinski R, Mackman N, Rak J (2014) Tissue factor expression provokes escape from tumor dormancy and leads to genomic alterations. Proc Natl Acad Sci USA 111(9): 3544-3549.

McCloy RA, Rogers S, Caldon CE, Lorca T, Castro A, Burgess A (2014) Partial inhibition of Cdk1 in G 2 phase overrides the SAC and decouples mitotic events. Cell Cycle 13(9): 1400-1412.

Qian BZ, Li J, Zhang H, Kitamura T, Zhang J, Campion LR, Kaiser EA, Snyder LA, Pollard JW (2011) CCL2 recruits inflammatory monocytes to facilitate breast-tumour metastasis. Nature 475(7355): 222-225.

Roca H, Varsos ZS, Sud S, Craig MJ, Ying C, Pienta KJ (2009) CCL2 and interleukin-6 promote survival of human $\mathrm{CD} 11 \mathrm{~b}+$ peripheral blood mononuclear cells and induce M2-type macrophage polarization. J Biol Chem 284(49): 34342-34354.

Sansone P, Storci G, Tavolari S, Guarnieri T, Giovannini C, Taffurelli M, Ceccarelli C, Santini D, Paterini P, Marcu KB, Chieco P, Bonafe M (2007) IL-6 triggers malignant features in mammospheres from human ductal breast carcinoma and normal mammary gland. J Clin Invest 117(12): 3988-4002.

Siegel RL, Miller KD, Jemal A (2017) Cancer statistics, 2017. CA Cancer J Clin 67(1): 7-30.

Swift J, Ivanovska IL, Buxboim A, Harada T, Dingal PC, Pinter J, Pajerowski JD, Spinler KR, Shin JW, Tewari M, Rehfeldt F, Speicher DW, Discher DE (2013) Nuclear lamin-A scales with tissue stiffness and enhances matrix-directed differentiation. Science 341(6149): 1240104.

Tabariès S, Siegel P (2011) Breast cancer liver metastasis. In Liver Metastasis: Biology and Clinical Management, Brodt P (ed) Vol. 16, Chapter 10, pp 273-306. Springer: NY, USA.

Taylor DP, Clark A, Wheeler S, Wells A (2014) Hepatic nonparenchymal cells drive metastatic breast cancer outgrowth and partial epithelial to mesenchymal transition. Breast Cancer Res Treat 144(3): 551-560.

Taylor DP, Wells JZ, Savol A, Chennubhotla C, Wells A (2013) Modeling boundary conditions for balanced proliferation in metastatic latency. Clin Cancer Res 19(5): 1063-1070.

Wheeler SE, Clark AM, Taylor DP, Young CL, Pillai VC, Stolz DB, Venkataramanan R, Lauffenburger D, Griffith L, Wells A (2014) 
Spontaneous dormancy of metastatic breast cancer cells in an all human liver microphysiologic system. Br J Cancer 111(12): 2342-2350.

Xu L, Hui AY, Albanis E, Arthur MJ, O’Byrne SM, Blaner WS, Mukherjee P, Friedman SL, Eng FJ (2005) Human hepatic stellate cell lines, LX-1 and LX-2: new tools for analysis of hepatic fibrosis. Gut 54(1): 142-151.

Yang M, Ma B, Shao H, Clark AM, Wells A (2016) Macrophage phenotypic subtypes diametrically regulate epithelial-mesenchymal plasticity in breast cancer cells. BMC Cancer 16(1): 419.

Yao C, Lin Y, Chua MS, Ye CS, Bi J, Li W, Zhu YF, Wang SM (2007) Interleukin-8 modulates growth and invasiveness of estrogen receptornegative breast cancer cells. Int J Cancer 121(9): 1949-1957.

Yuan N, Meng M, Liu C, Feng L, Hou L, Ning Q, Xin G, Pei L, Gu S, Li X, Zhao X (2014) Clinical characteristics and prognostic analysis of triple-negative breast cancer patients. Mol Clin Oncol 2(2): $245-251$.

Zhao W, Zhang L, Yin Z, Su W, Ren G, Zhou C, You J, Fan J, Wang X (2011) Activated hepatic stellate cells promote hepatocellular carcinoma development in immunocompetent mice. Int J Cancer 129(11): 2651-2661.

This work is published under the standard license to publish agreement. After 12 months the work will become freely available and the license terms will switch to a Creative Commons AttributionNonCommercial-Share Alike 4.0 Unported License.

Supplementary Information accompanies this paper on British Journal of Cancer website (http://www.nature.com/bjc) 